\title{
Supermode spatial solitons via competing nonlocal nonlinearities
}

\author{
Pawel S. Jung, ${ }^{* 1,2}$ Mirosław A. Karpierz, ${ }^{1}$ Marek Trippenbach, ${ }^{3}$ Demetrios N. Christodoulides, ${ }^{2}$ \\ and Wieslaw Krolikowski ${ }^{4,5}$ \\ ${ }^{1}$ Faculty of Physics, Warsaw University of Technology, Koszykowa 75, 00-662 Warsaw, Poland, \\ ${ }^{2}$ CREOL, The College of Optics and Photonics, University of Central Florida, Orlando, FL 32816, USA \\ ${ }^{3}$ Faculty of Physics, University of Warsaw, Pasteura 7, PL-00-681 Warszawa, Poland, \\ ${ }^{4}$ Laser Physics Centre, Research School of Physics and Engineering, Australian National University, Canberra, \\ ACT 0200, Australia \\ ${ }^{5}$ Science Program, Texas A\&M University at Qatar, Doha, Qatar
}

Received June 14, 2018; accepted June 25, 2018; published June 30, 2018

\begin{abstract}
We study spatial soliton formation in a system with competing nonlinearities. In doing so, we consider a specific nonlinear response that involves both focusing and defocusing nonlocal contributions. We demonstrate that at a sufficiently high input power level, the interplay between these nonlocal nonlinearities may lead to the formation of in-phase, two-hump, fundamental spatial solitons. The conditions required for the existence of these two-peak spatial solitons are also presented.
\end{abstract}

Spatial optical solitons are beams that propagate without spreading in nonlinear media due to a balance between diffraction and self-focusing. Over the years, they have been observed and studied within the context of various nonlinear media with spatially local, nonlocal, Kerr-like, and saturable nonlinearities [1-5]. The growing interest in materials having spatially nonlocal nonlinearities stems from their ability in arresting the catastrophic collapse of finite size beams supporting the formation of stable solitons [6-11]. In general, fundamental solitons in any type of media, exhibit as a single peak or a bell-like shape. However, we have recently shown that the competition between nonlocal nonlinearities may lead to fundamental solitons with two peaks, otherwise called super-mode solitons [12-13]. We theoretically demonstrated the existence of such solitons in planar, nematic liquid crystal configurations. In such systems reorientational focusing nonlinearity is accompanied by thermally-mediated defocusing. Interestingly, in liquid crystal environments, the competition between these types of nonlinearities introduces two oppositely acting interactions through a multiplicative model.

In this Letter, we consider the formation of multi-peak solitons in a simpler, and more general additive model for competing nonlocal nonlinearities, which could be applicable to a wider class of materials. We will also analytically present the necessary conditions these materials should satisfy for supporting this class of twohump solitons. a)

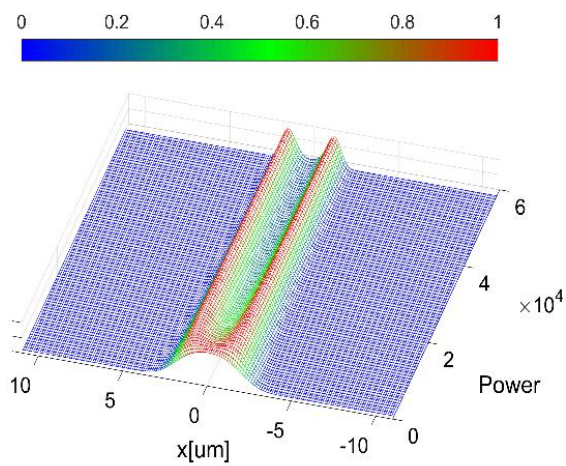

b)

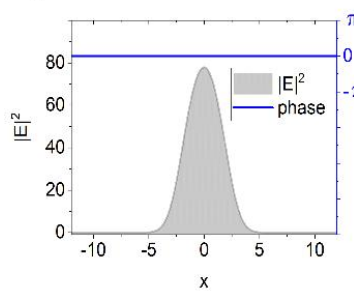

c)

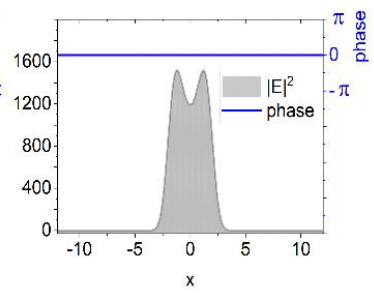

Fig. 1. (a) Normalized soliton solutions in the model of Eq. (1) with competing focusing and defocusing nonlocal nonlinearities as a function of beam power. Spatial intensity (grey area) and soliton phase (solid blue line) profiles for various normalized power levels are shown in b) $P=1 \cdot 10^{3}$, c) $P=5 \cdot 10^{3}$. In our simulations $\gamma=0.6, \sigma_{\alpha}=5, \sigma_{\beta}=4$.

We start by considering a scaled one-dimensional nonlinear Schrödinger equation that describes the nonlinear beam propagation of the electric field amplitude $\mathrm{E}(\mathrm{x}, \mathrm{z})$ :

$$
2 i \frac{\partial}{\partial z} E=\frac{\partial^{2}}{\partial x^{2}} E+f\left(|E|^{2}\right) \mathrm{E},
$$

where the nonlinearity of the medium $f\left(|E|^{2}\right)$ has the following form $f\left(|E|^{2}\right)=\alpha\left(|E|^{2}\right)-\beta\left(|E|^{2}\right)$, where the self-focusing function $\alpha\left(|E|^{2}\right)$ of the intensity $|E|^{2}$, is given by:

$$
\alpha\left(|E|^{2}\right)=\int_{-\infty}^{+\infty} R_{\alpha}\left(x^{\prime}-x\right)\left|E\left(x^{\prime}\right)\right|^{2} d x^{\prime},
$$

*E-mail: jung@if.pw.edu.pl 
while the nonlocal defocusing $\beta\left(|E|^{2}\right)$ contributions are described by:

$$
\beta\left(|E|^{2}\right)=\gamma \int_{-\infty}^{+\infty} R_{\beta}\left(x^{\prime}-x\right)\left|E\left(x^{\prime}\right)\right|^{2} d x^{\prime} .
$$

Here $\gamma$ is the relative strength of defocusing nonlinearity and the response functions $R_{\alpha, \beta}=\frac{1}{\sqrt{\pi} \sigma_{\alpha, \beta}} e^{-\frac{x^{2}}{\sigma_{\alpha, \beta}^{2}}}$ are Gaussian with the unit norm $\int_{-\infty}^{+\infty} R_{\alpha, \beta} d x=1$. The spatial extent (degree of nonlocality) of focusing and defocusing nonlinearities is represented by $\sigma_{\alpha}$ and $\sigma_{\beta}$, respectively.

The stationary bright soliton solutions of the system of Eqs. (1-3) were then obtained numerically using finite differences and imaginary time methods [14]. To test the stability of these solitons we used Finite Differences Beam Propagation Methods (FD-BPM) involving the Runge-Kutta $4^{\text {th }}$ order algorithm and evanescent field boundary conditions [15] so as to eliminate any reflections from the computational window. These results were also tested independently by employing split step Fast Fourier Transform methods.

Our simulations indicate that this general additive model of competing nonlinearities supports the existence of bright spatial solitons and, in particular, super-mode solitons as long as the self-focusing process prevails. The corresponding soliton intensity profiles, are depicted in Fig. 1 , as a function of the total power $(P=$ $\left.\int_{-\infty}^{+\infty}|E(x)|^{2} d x\right)$. From here it is evident that for lower powers, the solitons are simply standard single-peak, belllike beams (Fig. 1b).These are formed in the regime where the focusing nonlinearity dominates. However, as the power increases, this medium starts to support the formation of two-peak solitons instead (Fig. 1c).

It should be stressed that despite exhibiting a two-peak structure these self-trapped beams are not a higher-order or a bound state of two out-of-phase solitons [7]. In fact, they represent fundamental solitons having a constant phase distribution (see Fig. 1c). Additional simulations indeed confirm that these entities are stable during propagation and can survive acute collisions with other solitons.

To understand better the phenomenon behind the formation of such multi-peak solitons one needs to analyze carefully the interplay between the focusing $\left(\alpha\left(|E|^{2}\right)\right)$ and defocusing $\left(\beta\left(|E|^{2}\right)\right)$ terms. In Fig. 2, we illustrate the focusing and defocusing contributions to the nonlinearity (along the total nonlinearity $f\left(|E|^{2}\right)$ ) for a single-hump and a two-peak soliton, for certain input parameters. As we can see, for low powers, the defocusing part $\left(\beta\left(|E|^{2}\right)\right)$ is not strong enough compared

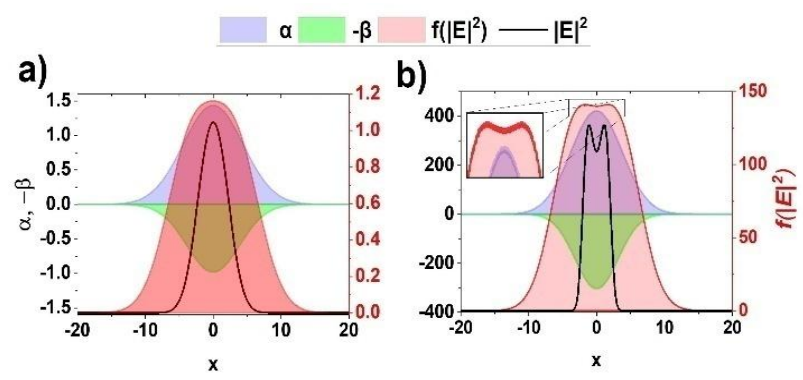

Fig. 2. Interplay between focusing $\left(\alpha\left(|E|^{2}\right)\right.$, blue area) and defocusing $\left(\beta\left(|E|^{2}\right)\right.$, green area) contributions to the nonlinear response $\left(f\left(|E|^{2}\right)\right.$, red area) for (a) single-peak and (b) two-peak soliton intensity distributions (indicated by the solid black line). Here $\gamma=0.6, \sigma_{\alpha}=$ $5, \sigma_{\beta}=4$.

with that from the focusing $\left(\alpha\left(|E|^{2}\right)\right)$ contribution (see Fig. 2a) and, as a result, the nonlinearity $f\left(|E|^{2}\right)$ has a smooth single peak profile supporting a standard, singlehump soliton. The situation changes dramatically when the power is increased. In this case, the nonlinearity profile starts to flatten at the centre. For even higher powers, the interplay between focusing $\left(\alpha\left(|E|^{2}\right)\right)$ and defocusing $\left(\beta\left(|E|^{2}\right)\right)$ leads to a more complex profile for the nonlinear response, having two maxima and thus supporting a two-hump soliton (see Fig. 2b). Using the waveguide analogy, these solitons are similar to the wellknown super-modes associated with linear and nonlinear waveguide arrays $[16,17]$. However, here a super-mode soliton represents the fundamental nonlinear mode of the self-induced complex waveguide structure.

Next, it is important to identify the conditions required in this additive model for the medium to support two-peak fundamental solitons. First, the overall nonlinearity must be positive $\left(f\left(|E|^{2}\right)>0\right)$, i.e. as a means to establish bright spatial solitons. If we assume, for simplicity the ansatz, that the soliton profile below and at the threshold can be represented by the Gaussian function $E=A e^{-\frac{x^{2}}{w^{2}}}$, then we arrive at the following condition:

$$
\left(1+2\left(\frac{\sigma_{\beta}}{w}\right)^{2}\right)>\gamma^{2}\left(1+2\left(\frac{\sigma_{\alpha}}{w}\right)^{2}\right) .
$$

Unfortunately, this relation involves the width $(w)$ that is unknown in our solution. If we further assume that the nonlocality is weak $\left(\sigma_{\alpha, \beta} \ll w\right)$, and by keeping in mind that we are in the focusing regime (Eq. (4)), we find that the strength of the defocusing nonlinearity should be in the range $0<\gamma<1$. If we now consider the opposite regime, i.e., a highly nonlocal case, such that $\left(\sigma_{\alpha, \beta} \gg w\right)$, from Eq. (4), we obtain:

$$
\sigma_{\beta}>\gamma \sigma_{\alpha}
$$


a)

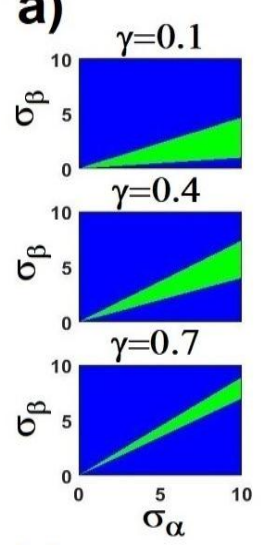

b)

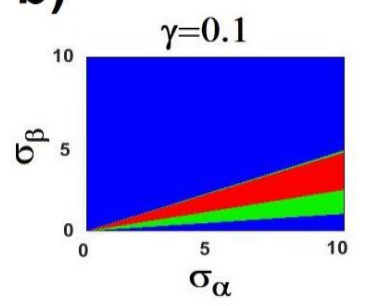

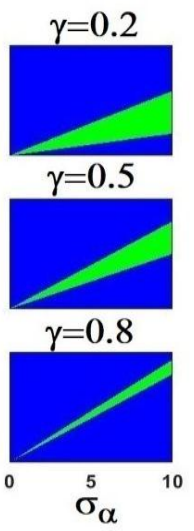

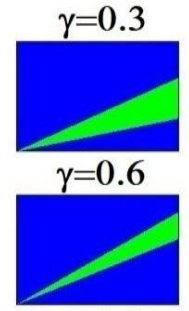

$\gamma=0.9$

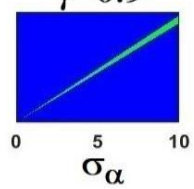

$\sigma_{\alpha}$

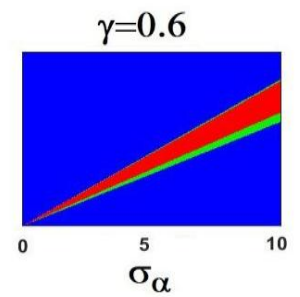

Fig. 3. (a) Analytically predicted $\left(\sigma_{\alpha}-\sigma_{\beta}\right)$ domain of existence of twopeak solitons, for different values of relative strength of defocusing nonlinearity $\gamma$. (b) Comparison of soliton existence domain at selected values of $\gamma$ found analytically (green) and numerically (red).

Finally, since we are interested in the appearance of two symmetrically placed light intensity peaks, the nonlinear index change is expected to exhibit a local minimum at $\mathrm{x}=0$, i.e. $\left.\frac{d f\left(|E|^{2}\right)}{d x}\right|_{x=0}>0$ [13]. For our additive model, this leads to the following relation:

$$
\sigma_{\beta}<\sqrt[3]{\gamma} \sigma_{\alpha}
$$

In Fig. 3a we demonstrate the domains where such twohump solitons exist in terms of nonlocality parameters $\left(\sigma_{\alpha}, \sigma_{\beta}\right)$ and relative strength of defocusing $\gamma$. The blue and green regions correspond to single and two-peak solitons, respectively. These results were obtained using both analytical and numerical techniques. In fact, we verified their accuracy using numerical simulations of the exact model Eq. (1), for various degrees of nonlocality and various strengths of nonlinearities. These results are shown in Fig. 3b, where we observe a good agreement between our simple analytical formulas and numerical simulations. Clearly, the existence region for these two peak solitons as predicted analytically, is smaller than that found numerically. Moreover, to describe a single peak splitting at the threshold, we looked for a splitting in the nonlinearity rather that a splitting in light intensity. This implies that, even though the nonlinear response profile already exhibits two humps, it still does not guarantee the appearance of two-hump solitons. Nevertheless, this analytical approach provides good (as confirmed numerically) predictions concerning system parameters supporting super-mode spatial solitons.

In conclusion, we studied theoretically the formation of fundamental bright solitons in media in the presence of additive competing nonlocal nonlinearities. To the best of our knowledge, we showed for the first time that stable two-hump super-mode spatial solitons exist in such a model. Additional simulations also indicate that this model supports not only two- but also multiple-peak solitons. Finally, we derived simple analytical formulas describing the existence domains of these two-peak solitons.

Pawel S. Jung thanks the Polish Ministry of Science and Higher Education for Mobility Plus scholarship. This work was support by Qatar National Research Fund (Grant \# NPRP 9-020-1-006) and Polish National Science Centre grant 2016/22/M/ST2/00261.

\section{References}

[1] G. Stegeman and M. Segev, Science 286, 1518 (1999)

[2] Y. Kivshar, G.P. Agrawal, Optical Solitons: From Fibers to Photonic Crystals (Academic, San Diego 2003).

[3] P. Varatharajah et al., Opt. Lett. 13, 690 (1988).

[4] G. Assanto, M. Peccianti, IEEE J. Quantum Electron. 39, 13 (2003).

[5] G. Assanto, ed. Nematicons: Spatial Optical Solitons in Nematic Liquid Crystals (Wiley, 2012).

[6] O. Bang, W. Krolikowski, J.Wyller, J.J. Rasmussen, Phys. Rev. E 66, 046619 (2002).

[7] X. Hutsebaut, C. Cambournac, M. Haelterman, A. Adamski, K. Neyts, Opt. Commun. 333, 211 (2004).

[8] C. Conti, M. Peccianti, G. Assanto, Phys. Rev. Lett. 91, 073901 (2003).

[9] U.A. Laudyn, P.S. Jung, M.A. Karpierz, G. Assanto, "Quasi twodimensional astigmatic solitons in soft chiral metastructures," Sci. Rep. 6, 22923 (2016).

[10] U.A. Laudyn, P.S. Jung, M.A. Karpierz, G. Assanto, Opt. Lett. 39(22), 6399 (2014).

[11] Y.V. Izdebskaya, V.G. Shvedov, P.S. Jung, W. Krolikowski, Opt. Lett. 43, 66 (2018).

[12] P.S. Jung, W. Krolikowski, U.A. Laudyn, M. Trippenbach and M.A. Karpierz, Phys. Rev. A 95, 023820 (2017).

[13] P.S. Jung, W. Krolikowski, U.A. Laudyn, M.A. Karpierz, M. Trippenbach, Opt. Expr. 25, 23893 (2017).

[14] S. Jungling, J.C. Chen, IEEE J. Quantum Electron. 30, 2098 (1994).

[15] P.S. Jung, K. Rutkowska, M.A. Karpierz, J. Comp. Science 25, 115 (2018).

[16] A.A. Hardy, W. Streifer, IEEE J. Lightwave Techn. LT-3, 1135 (1985)

[17] M. Matuszewski, B.A. Malomed, M. Trippenbach, Phys. Rev. A 75, 063621 (2007). 